\title{
Improving communication of inpatient blood transfusion events to GPs
}

\author{
Timothy Robbins
}

University Hospitals Coventry and Warwickshire NHS Trust

\begin{abstract}
Patients who have had blood transfusions whilst in hospital must have this information communicated to their General Practitioner at discharge. Audit demonstrated that just $50 \%$ of patients $(n=15)$ under medical specialties who had undergone a blood transfusion had this information included in their discharge letter. To improve this, a section was specifically designated on the e-discharge pro-forma for the documentation of blood transfusion events, and focused teaching was delivered to all new FY1 doctors at their induction. Post intervention, $80 \%$ of blood transfusions occurring in medical patients were documented on the e-discharge, with an improvement in how detailed this documentation was $(n=40)$. This simple intervention is an easily reproducible, cost neutral method of ensuring that more blood transfusion events are communicated to patients' GPs; improving care and reducing risk.
\end{abstract}

\section{Problem}

Junior doctors are often not aware of, or forget about the need to include inpatient blood transfusion information on discharge letters to general practitioners. This information is of significant benefit to GPs as it enables a better understanding of the inpatient admission, facilitates more informed discussions with patients about their admission, and can influence post-admission care. Knowledge of transfusion events also allows GPs to manage post-transfusion care (eg haemogolobin monitoring), as well as identifying potential complications, which can be particularly important if the transfusion is completed close to the time of discharge. With a trend towards shorter hospital admissions it is increasingly likely that GPs will be seeing patients soon after a transfusion, thus increasing the importance of this project.

This project was completed in University Hospitals Coventry and Warwickshire NHS Trust - a 1250 bed tertiary referral centre in the West Midlands, England.

\section{Background}

Concerns regarding the quality of discharge letters to GPs are longstanding, and the communication of blood transfusion events specifically highlighted. (1) The importance of communicating blood transfusion events on discharge letters features in many local guidelines and is realised at a national level. (2) There is however little data in the research literature regarding this issue. The use of specific templates has been shown to improve the quality of edischarge letters in other specialties. (3) Ideally the increasing use of IT systems would allow the transfusion of a patient to be automatically included in e-discharge letters following the electronic recording of the fate of blood products, however incompatibilities between IT packages make this impossible in many Trusts, and solutions are certainly needed in the short-medium term.

\section{Baseline Measurement}

A retrospective audit was completed studying patients who were treated by medical specialties at University Hospital Coventry and Warwickshire NHS Trust between May and July 2012 and who received a blood transfusion. Patients under the care of haematology and oncology were excluded due to differing transfusion policies and reporting arrangements. All patients who received a red blood cell transfusion during this period were identified from the BloodTrack database. 15 patients were randomly selected using Microsoft Excel random number generator, and their e-discharges reviewed to identify whether the inpatient blood transfusion event had been recorded. Results showed that $50 \%$ of edischarges identified that the patient had received a blood transfusion. Of those that identified whether a transfusion occurred, $13 \%$ recorded on what date the transfusion occurred, $40 \%$ whether or not a transfusion reaction occurred, $82 \%$ how many units had been transfused, and $86 \%$ why the transfusion occurred. $69 \%$ of all e-discharge letters were completed by F1/F2 doctors.

\section{Design}

We designed an intervention whereby a box on the e-discharge proforma was specifically labelled for the inclusion of blood transfusion information. This box allowed space for free text entry, and a separate space for writing the date the transfusion occurred. Multiple blood transfusions could be recorded. This change was incorporated into the hospital IT system on the 27th June 2013.

Additionally having noted the high proportion of discharge letters that were completed by FY1/FY2 doctors, we arranged for the blood transfusion matrons to teach the incoming FY1s about the importance of including blood transfusion events on the e-discharge letters. This occurred within an already scheduled teaching session with the blood transfusion team and the new FY1 doctors.

This design is fully sustainable; the inclusion of the section on blood transfusions in the e-discharge proforma is a permanent change. Furthermore all new F1s to the Trust already receive teaching from the blood transfusion team, and specific information on discharge letters will now feature as part of this in the future. 


\section{Strategy}

The initial plan was to design a system whereby the BloodTrack database, which records the fate of all blood products in the Trust, would automatically link to the patient records system with blood transfusions events, then automatically being included in the edischarge template. This however was not possible due to software incompatibilities. Whilst University Hospitals Coventry and Warwickshire NHS Trust uses a bespoke electronic patients record system designed in-house, our IT department report that this is not an isolated problem and it is likely that other Acute NHS Trusts would have similar problems. We therefore modified our intervention to that described above, and whilst there was significant negotiations with IT, this was accepted and rolled out hospital wide on the 27th June 2013.

\section{Results}

The retrospective audit was repeated following the intervention. Patients who were treated by medical specialties at University Hospital Coventry and Warwickshire NHS Trust during August 2013 and who received a blood transfusion were included. Patients treated by haematology and onclogy were excluded due to differing transfusion policies and reporting arrangements. All patients who received a red blood cell blood transfusion during this period were identified from the BloodTrack database. 40 patients were randomly selected using Microsoft Excel random number generator and their e-discharges reviewed to identify whether the inpatient blood transfusion event had been included. Results showed that $80 \%$ of edischarges identified that the patient had received a blood transfusion (compared to $50 \%$ pre-intervention). Of those that identified whether a transfusion had occurred, 50\% recorded on what date the transfusion occurred (compared to $13 \%$ preintervention), $56 \%$ whether or not a transfusion reaction occurred (compared to $40 \%$ pre-intervention), $97 \%$ how many units had been transfused (compared to $82 \%$ pre-intervention), and $91 \%$ why the transfusion occurred (compared to $86 \%$ pre-intervention).

\section{Lessons and Limitations}

Perhaps the most important learning point is the impact such a simple intervention can have. The process of allocating a particular space for information to be included in a pro-forma alongside the topic being included in a pre-existing teaching session achieved a significant benefit, was cost neutral and has long term sustainability. It demonstrates that we should regularly review our pro-formas to identify whether alternative/additional information should be included. There is a further lesson about the restrictive nature of healthcare IT systems in integrating with one another but also the ability to work solutions around this.

Limitations of the study include the limited follow-up time before reaudit, however the recent requirement for consent for blood transfusions means that it would be difficult to separate whether this has a knock-on benefit to improving communication with general practitioners if the audit was repeated later. A further limitation is that this study was isolated to medical specialty patients only and the benefits could have been explored for the other specialties, though the benefits are expected to be similar.

\section{Conclusion}

The communication of inpatient blood transfusion events to General Practitioners can be improved by including a dedicated space for this information on e-discharge proformas and by including the importance of completing such a section in junior doctor teaching. This is a cost-neutral sustainable solution which can be applied when it is not possible to co-ordinate blood tracking software to edischarge/patient record software.

\section{References}

1. GPs flag concerns on discharge delays. PULSE; 2012

2. Serious Hazards of Transfusion (SHOT) scheme (1996-2011) SHOT Annual Reports 1996 - 2011. SHOT Office, Manchester.

3. Taylor C: Improving e-discharge letters for Permanent pacemaker insertion at Wansbeck General Hospital. BMJ Qual Improv Report 2013 Aug 27; 2

\section{Declaration of interests}

Nothing to declare

\section{Acknowledgements}

Particular thanks to the Blood Transfusion team at University Hospitals Coventry and Warwickshire NHS Trust, in particular Mrs Janine Beddow (Transfusion Matron). 